\title{
EDITORIAL
}

\section{Focus on imaging in trauma}

\author{
Frank Hildebrand ${ }^{1} \cdot$ K. Eichler ${ }^{2}$
}

Published online: 31 January 2018

c) Springer-Verlag GmbH Germany, part of Springer Nature 2018

Modern emergency room management requires interdisciplinary teamwork and synchronous communication between a team of surgeons, anesthetists, and radiologists. With the direct involvement of modern imaging techniques, traumarelated injuries should be detected within a very short period of time to enable a priority-orientated treatment. However, also the potential harms of CT (e.g., risk of radiationinduced cancer, increased costs, or overdiagnosis) particularly after minor or moderate trauma have to be considered.

To underline the importance of imaging in trauma patients, this issue of the European Journal of trauma and Emergency Surgery is focused on recent topics.

In the first paper, Grünherz et al. [1] performed an online cross-sectional survey with nine online questions and assessed the opinion of trauma surgeons about the early use of whole-body computed tomography (WBCT) in stable and unstable patients. For hemodynamically stable patients, the vast majority of trauma surgeons recommended "focused assessment with sonography in trauma" (FAST) and early $\mathrm{CT}$ as the best diagnostic tools. In hemodynamically unstable patients more than $90 \%$ considered FAST as mandatory, whereas only less than half of the trauma surgeons $(47.5 \%)$ considered CT to be essential. Therefore, it appears that the acceptance of early WBCT in hemodynamically unstable patients still seems to be limited. Based on their findings, the authors suggest the optimization of local circumstances in each hospital to enable WBCT during emergency room management in all severely injured patients regardless of their hemodynamic status.

In conscious and hemodynamically stable blunt trauma patients (mean ISS 8), Moussavi et al. [2] prospectively

K. Eichler

k.eichler@em.uni-frankfurt.de

1 Department of Trauma and Reconstructive Surgery, University Hospital Aachen, Pauwelsstrasse 30, 52074 Aachen, Germany

2 Institute of Diagnostic and Interventional Radiology, University Hospital Frankfurt, Theodor-Stern Kai 7, 60590 Frankfurt, Germany assessed the relevance of performing routine versus selective (i.e., according to clinical presentation) chest and abdominalpelvic CT scan for hospitalization time and outcome after blunt trauma. In this study, a two-to-threefold increase of CT scans after routine $\mathrm{CT}$ utilization was observed. The routine approach was also associated with an increased detection of unexpected injuries and a significant reduction of hospitalization time that is mainly due to a shorter time to diagnosis and treatment. However, no effect on the incidence of posttraumatic complications and outcome was found. The authors concluded that the selective approach of CT scanning has the potential to reduce radiation exposure, costs as well as overuse and should be investigated in further studies. Due to the low mean injury severity of the included patients, these conclusions can only be applied to patients with mildto-(at best) moderate trauma severity.

The third article by Onwubiko et al. [3] focused on incidental findings after $\mathrm{CT}$ of the abdomen and pelvis in pediatric trauma patients. In an analysis of their level I pediatric trauma center registry, records were reviewed for any incidental findings that were not related to the initial injuries. The overall study population of 241 patients revealed 114 incidental findings in 86 patients. 47 incidental findings were of potential clinical significance. Seven patients had a further workup of the diagnosis, and three underwent a surgical intervention. The authors concluded that although a significant number of incidental findings were anatomic variants with no diagnostic or therapeutic relevance, a good communication between the trauma team and other specialists is essential to adequately workup the findings with potential clinical significance.

To quantify how often CT scans contribute to the diagnosis of relevant injuries, Hansen et al. [4] applied the "Negative CT score". This score is calculated subtracting the number of body regions with a relevant positive CT finding from the total number of scanned body regions. The four body regions used to calculate the "Negative CT score" are head, neck, chest, and abdomen/pelvis. In their retrospective study including patients with mild-to-moderate trauma severity (mean ISS 7), an average number of 2.36 body regions were 
scanned per patient. The mean "Negative CT score" was 2.10 which mean that on average, two body regions were scanned without important findings. To optimize the posttraumatic utilization of CT scans, this score might help to develop and compare protocols for CT imaging within and across trauma centers.

In a retrospective study of Kritsaneepaiboon et al. [5], the association between cumulative radiation exposure and the lifetime attributable risk (LAR) of radiation-induced cancer after CT of adult multiple trauma patients was investigated. They found an average cumulative dose of $19.4 \mathrm{mSv}$ per patient and an LAR of cancer of $0.14 \%$. Young patients with an age under 30 years, females, and multiple or repeated CT scans of the trunk exhibited the highest risk of malignant diseases.

The presented articles reflect the current imaging procedure in trauma patients as well as limitations, problems, and challenges for the future.

We hope you enjoy reading our selection of topics around the imaging of trauma patients.

\section{Compliance with ethical standards}

Research involving human and animal participants The authors comply with the ethical guidelines for authorship and publishing the European Journal of Trauma and Emergency Surgery. This article does not certain any studies with human or animal subjects performed by the authors.
Conflict of interest Katrin Eichler and Frank Hildebrand declare no conflict of interest in relation with this work.

\section{References}

1. Grünherz L, Jensen KO, Neuhaus V, Mica L, Werner CML, Ciritsis B, Michelitsch C, Osterhoff G, Simmen HP, Sprengel K. Early computed tomography or focused assessment with sonography in abdominal trauma: what are theleading opinions? Eur J Trauma Emerg Surg. 2017. https://doi.org/10.1007/s00068-017-0816-4.

2. Moussavi N, Ghani H, Davoodabadi A,. Atoof F, Moravveji A, Saidfar S, Talari H. Routine versus selective chest and abdominopelvic CT-scan in conscious blunt trauma patients: arandomized controlled study. Eur J Trauma Emerg Surg. 2017. https://doi. org/10.1007/s00068-017-0842-2.

3. Onwubiko C, Mooney DP. The prevalence of incidental findings on computed tomography of the abdomen/pelvis in pediatric traumapatients. Eur J Trauma Emerg Surg. 2017. https://doi. org/10.1007/s00068-017-0798-2.

4. Hansen CK, Strayer RJ, Shy BD, Kessler S, Givre S, Shah KH. Prevalence of negative CT scans in a level one trauma center. Eur J Trauma Emerg. 2016. https://doi.org/10.1007/s00068-016-0741-y.

5. Kritsaneepaiboon S, Jutiyon A, Krisanachinda A. Cumulative radiation exposure and estimated lifetime cancer risk in multiple injury adult patients undergoing repeated or multiple CTs. Eur J Trauma Emerg Surg. 2016. https://doi.org/10.1007/s0006 8-016-0665-6. 